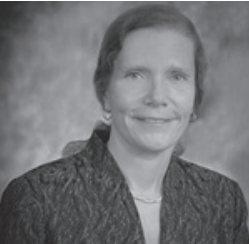

Michele Upvall, PhD, $\mathrm{RN}, \mathrm{CNE}, \mathrm{FAAN}$, is a Professor of Nursing and Program Director of the Nurse Educator program at the University of Central Florida in Orlando, Florida. She is co-editor of Global Health Nursing: Building and Sustaining Partnerships.

\section{Moral Agency in the Context of Global Volunteering}

Michele Upvall, PhD, RN, CNE, FAAN

Marcia Sue DeWolf Bosek, DNSc, RN

Martha Turner, PhD, RN-BC, FAAN, Col., USAF NC (ret)

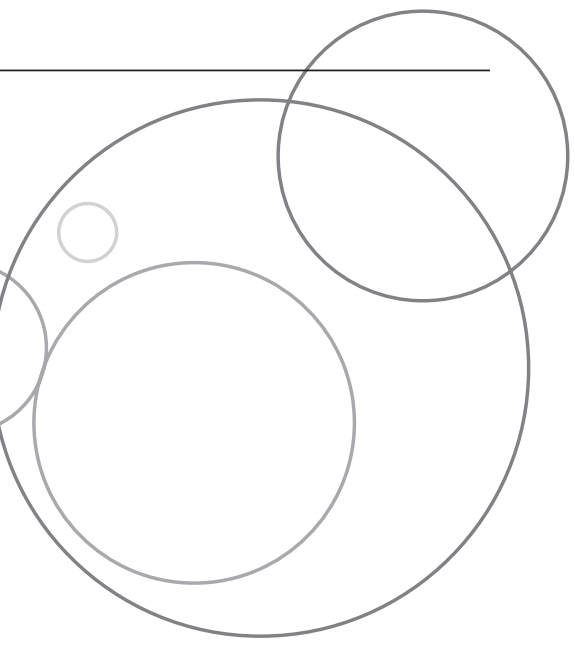

Today there are many global volunteer opportunities for nurses and student nurses. While the intentions of volunteers may be good, the result may be harmful to the volunteer and/or the host, creating a situation of moral distress. An ethical code provides guidance and promotes moral agency. We discuss elements of the American Nurses Association Code of Ethics with Interpretive Statements and apply them to the experience of global volunteering through a case study approach.

Keywords: ethics; moral agency; global perspective; volunteering

A caring professionals, nurses often express their desire to serve others across the globe as volunteers. Pre-licensure and graduate nursing students are also eager to serve despite educational and employment obligations, and may seek short-term global volunteer experiences through schools (study abroad or service leaning) and churches (medical missions). These experiences offer many benefits to the volunteer, including increased cultural awareness, knowledge of nursing as a global profession, and an opportunity to "give back" or share their knowledge and skills with others (Lasker, 2016; Napolitano \& Duhamel, 2017).

Global volunteering can be a life-changing event, but nurses must be prepared to confront a host of challenges throughout the experience, from pre-departure planning to adjusting to the new environment and integrating lessons learned from the experience upon their return home. Ethical issues may emerge at any point in the volunteer experience, and pose a risk to both the volunteer and the host community. For example, the needs of the community may not be fully understood, especially when intercultural communication is not considered and/or misunderstandings due to breakdowns in communication occur (White \& Evert, 2014). There may also be conflicting expectations regarding host supervision, with the possibility of both pre-licensure students and experienced nurse volunteers working above their current skill level and/or scope of practice. While the standards and scope of practice may vary across countries, a working knowledge of the Code of Ethics for Nurses with Interpretive Statements (the Code; American Nurses Association [ANA], 2015) can provide a framework for nurse volunteers when uncertainty occurs or questions arise 
regarding scope of practice. The International Council of Nurses (2012) is another resource to guide the practice of both nurse volunteers and nurses from the host community.

Our intent is to highlight the ethical challenges confronted by volunteers in global settings, and to suggest strategies to reduce moral distress by strengthening their moral agency. We present a case study and explore how the nurse volunteer can respond as a moral agent living the ANA Code of Ethics for Nurses in a global setting.

\section{MORAL AGENCY AND DUTY TO SELF}

Moral agency, or the capacity to bring about good, is required for any volunteer experience, mediating the potential for moral distress (Grace, 2018; Jurchak et al., 2017). Keys to developing moral agency include cultivating personal and professional characteristics, and the active application of ethical knowledge for change (Jurchak et al., 2017). Although the entire Code, all nine provisions with their interpretive statements, offers a framework to develop moral agency, we selected specific interpretive statements (IS) to illustrate the ethical challenges encountered as a global volunteer (ANA, 2015).

The Code begins by articulating a fundamental principle, ".... respect for the inherent dignity, worth, unique attributes, and human rights of all individuals" (p. 1), and asserting that the right to health care is universal (ANA, 2015). The Code goes on to say in IS 2.1, "The nurse's primary commitment is to the recipients of nursing and healthcare services-patient or client-whether individuals, families, groups, communities, or populations" (p. 5).

Regardless of where the nurse volunteers, the Code emphasizes the nurse's responsibility toward those receiving care. However, volunteering is not an act of all-encompassing self-sacrifice. Nurses who volunteer must extend the same consideration to self as they extend to those in host communities.

Provision 5 of the Code clearly states, "The nurse owes the same duties to self as to others, including the responsibility to promote health and safety, preserve wholeness of character and integrity, maintain competence, and continue personal and professional growth" (p. 19). Nurse volunteers must first think of the duty they have to self before extending expertise to others. Respecting ourselves is paramount before we can extend respect and dignity to others. Mutuality, one of the pillars of global volunteering (Lasker, 2016), begins with honoring our own gifts brought to the volunteer experience and in turn, honoring and respecting the gifts of the host community members. Gifts in this context include the knowledge we give and gain from others as well as the affective component of the experience-the sharing of fears, frustrations, and joy inherent in volunteering. The Code further supports volunteer-host relationships, stating, "Nurses maintain professional, respectful, and caring relationships with colleagues" (ANA, 2015, p. 4); for nurse volunteers, this includes relationships with those from the host community. IS 2.3 provides further detail for developing relationships within the host community: "Collaboration intrinsically requires mutual trust, recognition, respect, transparency, shared decision-making, and open communication among all who share concern and responsibility for health outcomes" (p. 5).

Provision 5 of the Code also requires the nurse to be a model for health promotion and to promote safety and well-being, striving for balance in the care of self. IS 5.3 speaks to preservation of character, and suggests integration of personal and professional values into relationships. Authenticity in the relationship is important, but if

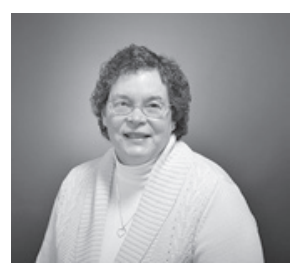

Marcia Sue DeWolf Bosek, DNSc, RN, is an Associate Professor of Nursing at the University of Vermont in Burlington, Vermont, and a Nurse Scientist at the University of Vermont Medical Center. She has more than twenty years of experience as a clinical ethicist.

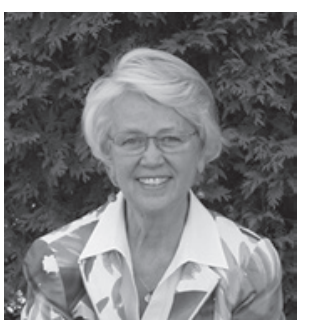

Martha Turner PhD, RN-BC, FAAN, Col., USAF NC (ret) is a consultant, lecturer, and author in Nursing Ethics, Nursing Leadership, Nursing Practice and Global Health. 
Ethical issues

may emerge at

any point in the

volunteer experi-

ence, and pose

a risk to both the

volunteer and the

host community.

there are conflicts in values, the nurse is required to "...render compassionate, respectful, and competent care" (ANA, 2015, p. 20). Volunteer nurses can expect differences in beliefs and values, and should be prepared for culture shock. Cultural differences may be more obvious when volunteering in a country or part of the world in which the prevalent values contrast sharply with the volunteer nurse's (White \& Evert, 2014). The introduction to the Code reminds the nurse that regardless of location,

Nurses establish relationships of trust and provide nursing services according to need, setting aside any bias or prejudice. Factors such as culture, value systems, religious or spiritual beliefs, lifestyle, social support system, sexual orientation or gender expression, and primary language are to be considered when planning individual, family and population-centered care. (ANA, 2015, p. 1)

Culture shock, and situations in which values conflict, may occur prior to, during, or after the volunteer experience, and can often lead to moral distress. IS 5.4 suggests a method to mitigate this distress: "Nurses have a right and a duty to act according to their personal and professional values and to accept compromise only if reaching a compromise preserves the nurse's moral integrity and does not jeopardize the dignity or well-being of the nurse or others" (ANA, 2015, p. 20). Again, respect is key, as the experience of mutuality promotes dialogue, fosters compromise, and facilitates conflict resolution.

Finally, IS 5.5 (ANA, 2015, p. 22) proposes that duty to self includes lifelong learning, maintaining professional competence, and continuation of personal growth. The nurse volunteer must first attend to new knowledge and integrate it into practice before extending knowledge to others. To do good and extend moral agency, the nurse volunteer must be committed to receiving new knowledge throughout the volunteer experience. In IS 5.6, we see the link between professional and personal growth: "Professional and personal growth reciprocate and interact. Activities that broaden nurses' understanding of the world and of themselves affect their understanding of patients; those that increase and broaden nurses' understanding of nursing's science and art, values, ethics, and policies also affect nurses' self- understanding" (ANA, 2015, p. 22).

\section{CASE STUDY: SHOULD I VOLUNTEER?}

The following case study is an opportunity for the nurse volunteer to apply guidance from the Code of Ethics for Nurses with Interpretive Statements (ANA, 2015) to an ethical encounter:

Maria, a new staff nurse on an orthopedic unit, approaches a colleague with extensive global volunteer experience, stating, "As my clinical mentor, I was hoping you would help me think through an opportunity that I'm considering. A group from my neighborhood community has asked me to participate in a short-term humanitarian trip since I'm a bilingual nurse. I'm excited about the possibility of participating in a variety of health clinics and even working in labor and delivery! This trip seems wonderful, but I've been reading on the internet that these trips can be controversial. What advice do you have?"

Maria's colleague knows that there are many things to consider when deciding to volunteer. Pragmatic considerations include financial costs, housing and travel arrangements, and arranging time away from work and home responsibilities. However, ethical considerations require deeper reflection than the more readily addressed practical concerns. 
When considering a volunteer opportunity, Maria's ethical commitment should be to benefit and not harm the host community. Specifically, she should evaluate how the short-term trip fits into a sustainable health-care plan for the host community. Without a sustainable plan, a short-term trip could create fluctuations in available medications, resources, and/or providers trained to use donated equipment, which could be detrimental to the community in the long run.

In addition, Maria should consider whether the presence of volunteers interrupts the social dynamics of the community. For example, would volunteering to rebuild a school take away jobs (and income) from local workers? Would Maria's presence further tax scarce resources such as clean water or food? If the organization plans to bring in a scarce resource for their own use, such as drinking water, would this action create an inequity between Maria and the host community that might harm the development of trust?

Once Maria has determined that the volunteer opportunity is ethically designed, she should also consider potential harms to self. Remembering that "the nurse owes the same duty to self as others..." (ANA, 2015, p. 19), Maria must weigh personal health and safety concerns when deciding whether to volunteer. Some considerations are logistical in nature. For example, if the country has a high incidence of malaria, will mosquito netting be provided or does the volunteer need to bring a net? Maria should also consider carefully the potential health risks associated with the planned activities. For example, if she has asthma, she may decide not to volunteer after learning that the planned activities involved removing flood-damaged drywall and cleaning areas that are tainted with mold and mildew. As with any overseas travel, all of Maria's vaccinations should be up to date. Finally, she should consider what actions would be appropriate if she becomes injured or ill. For example, if she broke a leg, how would this injury be treated? Would she return home before the end of the trip?

When volunteering, the temptation is strong to want to be involved in every activity in which the needs are great. IS 4.1 stipulates that "Nurses bear the primary responsibility for the nursing care that their patients and clients receive and are accountable for their own practice.... Nurses must always comply with and adhere to state nurse practice acts, regulations, standards of care and the ANA's Code of Ethics" (p. 15). Thus, Maria, an orthopedic nurse, would need to review her nursing skills and competencies to determine in advance what role(s) she could competently fulfill when volunteering on the labor and delivery unit. Regulations governing nursing in the host country would also need to be considered.

While volunteering, Maria would want to pay attention to behaving in a culturally sensitive manner. Being bilingual would promote Maria's ability to communicate and learn about the culture. Before leaving, she should investigate cultural norms for the host community. What social roles exist between men and women? Is it socially acceptable for women to wear pants? How are elders viewed?

Maria should also safeguard privacy and confidentiality when using social media. IS 3.1 states: "Nurses must maintain vigilance regarding postings, images, recordings, or commentary that intentionally or unintentionally breaches their obligation to maintain and protect patients' rights to privacy and confidentiality" (ANA, 2015, p. 9).

Other organizations have also commented on the sensitivity of photography while volunteering. "Before posting a photograph, the volunteer should have a purpose for using the photograph, such as inspiring hope and understanding" (Unite for Sight, n.d.). Maria should avoid posting photographs that perpetuate the image of suffering, reinforce stereotypes, or portray the community member as a prop for a volunteer selfie. Also, Maria should seek protocols for ethical photography based
The potential volunteer should evaluate how the short-term trip fits into a sustainable health-care plan for the host

community, as well as whether the presence of volunteers interrupts the social dynamics of the community. 
Volunteers

should avoid

posting pho-

tographs that

perpetuate the

image of suf-

fering, reinforce

stereotypes, or

portray the com-

munity member

as a prop for a

volunteer selfie. on organizational and host community guidelines in order to preserve the dignity of those being photographed (Maltby, 2017).

Lastly, Maria may suffer moral distress when she recognizes the wide disparities and inequalities between her familiar standards of practice and standards of care and those of the host country. Maria, along with the other nurses, will need to prepare for moral distress and think of strategies to encourage resilience. They should reflect on the conflicts between their standards and the standards of others. IS 2.2 advises that when conflicts arise, practices should "promote the patient's best interests while preserving the professional integrity of the nurse and supporting interprofessional collaboration" (ANA, 2015, p. 5).

\section{CONCLUSION}

Nurses choosing to participate as volunteers in global settings must consider a multiplicity of ethically challenging factors, and work to develop their own moral agency, so they can both achieve good in the host communities and alleviate their own moral distress. Moral agency of the global volunteer need not stop when returning home. Beyond immediate efforts to alleviate suffering while in the host community, efforts can continue at home to identify and eliminate the root causes of the health problems encountered. We challenge the reader to think of additional ethical situations that can cause moral distress in a global volunteer setting, and apply the Code to strengthen moral agency.

\section{REFERENCES}

American Nurses Association. (2015). Code of ethics for nurses with interpretive statements. Silver Spring, MD: Nursebooks.org.

Grace, P. (2018). Enhancing nurse moral agency. OJIN: The Online Journal of Issues in Nursing, 23. Retrieved from http://ojin.nursingworld.org/MainMenuCategories/ANAMarketplace/ANAPeriodicals/OJIN/TableofContents/Vol-23-2018/No1-Jan-2018/ Enhancing-Nurse-Moral-Agency.html

International Council of Nurses. (2012). The ICN code of ethics for nurses. Retrieved from http:/ / www.icn.ch/who-we-are/code-of-ethics-for-nurses /

Jurchak, M., Grace, P. J., Lee, S. M., Willis, D. G., Zollfrank, A. A., \& Robinson, E. M. (2017). Developing abilities to navigate through the grey zones in complex environments: Nurses' reasons for applying to a clinical ethics residency for nurses. Journal of Nursing Scholarship, 49(4), 445-455. http:/ /dx.doi.org/10.1111/jnu.12297

Lasker, J. N. (2016). Hoping to help: The promises and pitfalls of global health volunteering. Ithaca, NY: Cornell University Press.

Maltby, H. J. (2017). Ethical photography while on study abroad. Nurse Educator, 42(3), 112-114. http:/ / dx.doi.org/10.1097/NNE.0000000000000328

Napolitano, N., \& Duhamel, K. V. (2017). Reflections on an innovative approach to studying abroad in nursing. Creative Nursing, 23(1), 53-57. http:/ / dx.doi.org/10.1891/1078-4535.23. 1.53

Unite for Sight. (n.d.). Ethics and photography in developing countries. Retrieved from https://www.uniteforsight.org/global-health-university/photography-ethics

White, M., \& Evert, J. (2014). Developing ethical awareness in global health: Four cases for medical educators. Developing World Bioethics, 14(3), 111-116. http://dx.doi.org/10.1111/ dewb.12000

Correspondence regarding this article should be directed to Michele Upvall, PhD, RN, CNE, FAAN, at michele. upvall@ucf.edu 